\title{
ADDITIVE FUNCTIONS ON SHIFTED PRIMES
}

\author{
P. D. T. A. ELLIOTT
}

\begin{abstract}
Best possible bounds are obtained for the concentration function of an additive arithmetic function on sequences of shifted primes.
\end{abstract}

A real-valued function $f$ defined on the positive integers is additive if it satisfies $f(r s)=f(r)+f(s)$ whenever $r$ and $s$ are coprime. Such functions are determined by their values on the prime-powers.

For additive arithmetic function $f$, let $C_{h}$ denote the frequency amongst the integers $n$ not exceeding $x$ of those for which $h<f(n) \leq h+1$. Estimates for $C_{h}$ that are uniform in $h, f$, and $x$ play a vital rôle in the study of the value distribution of additive functions. They can be employed to develop criteria necessary and sufficient that a suitably renormalised additive function possess a limiting distribution, as well as to elucidate the resulting limit law. They bear upon problems of algebraic nature, such as the product and quotient representation of rationals by rationals of a given type. In that context their quantitative aspect is important.

It is convenient to write $a \ll b$ uniformly in $\alpha$ if on the values of $\alpha$ being considered the functions $a, b$ satisfy $|a(\alpha)| \leq c b(\alpha)$ for some absolute constant $c$. When the uniformity is clear, I do not declare it.

Let

$$
W(x)=4+\min _{\lambda}\left(\lambda^{2}+\sum_{p \leq x} \frac{1}{p} \min (1,|f(p)-\lambda \log p|)^{2}\right),
$$

where the sum is taken over the prime numbers. Improving upon an earlier result of Halász, Ruzsa proved that $C_{h} \ll W(x)^{-1 / 2}$, uniformly in $h, f$, and $x \geq 2$ [9]. This result is best possible in the sense that for each of a wide class of additive functions there is a value of $h$ so that the inequality goes the other way.

From a number theoretical point of view it is desirable to possess analogs of Ruzsa's result in which the additive function $f$ is confined to a particular sequence of integers of arithmetic interest. In this announcement I consider shifted primes.

Let $a$ be a nonzero integer. Let $Q_{h}$ denote the frequency amongst the primes $p$ not exceeding $x$ of those for which $h<f(p+a) \leq h+1$.

Theorem 1. The estimate $Q_{h} \ll W(x)^{-1 / 2}$ holds uniformly in $h, f$, and $x \geq 2$.

If for an integer $N \geq 3$ we define $S_{h}$ to be the frequency amongst the primes

Received by the editors November 26, 1991. Presented at the 1992 Illinois Number Theory Conference, University of Illinois at Urbana-Champaign, April 3-4, 1992.

1991 Mathematics Subject Classification. Primary 11K65, 11L20, 11N37, 11N60, 11 N64. 
$p$ less than $N$ of those for which $h<f(N-p) \leq h+1$, and set

$$
Y(N)=4+\min _{\lambda}\left(\lambda^{2}+\sum_{\substack{p<N \\(p, N)=1}} \frac{1}{p} \min (1,|f(p)-\lambda \log p|)^{2}\right),
$$

then there is an analogous result.

Theorem 2. The estimate $S_{h} \ll Y(N)^{-1 / 2}$ holds uniformly in $h, f$, and $N \geq 3$.

The estimates given in these two theorems are of the same quality as Ruzsa's and again best possible. In particular, Theorem 1 improves the bound $Q_{h} \ll$ $W(x)^{-1 / 2}(\log W(x))^{2}$ of Timofeev [10]. If

$$
E(x)=4+\sum_{\substack{p \leq x \\ f(p) \neq 0}} \frac{1}{p}
$$

then Timofeev shows that the number of primes not exceeding $x$ for which $f(p+a)$ assumes any (particular) value is $\ll \pi(x) E(x)^{-1 / 2}(\log E(x))^{2}$. Employing the present Theorem 1, the logarithmic factor may be stripped from this bound. The improved inequality is then analogous to an estimate of Halász concerning additive functions on the natural numbers and, in a sense, best possible [8].

The concentration function estimate of Theorem 2 also has many applications, in particular, to the study of the value distribution of additive functions. These are new and of a new type. They involve not only the primes but also the length of the interval on which the additive function is considered. Thus the frequencies

$$
(\pi(N-1))^{-1} \sum_{\substack{p<N \\ f(N-p) \leq z}} 1
$$

possess a limiting distribution function as $N \rightarrow \infty$ if and only if the three series

$$
\sum_{|f(p)|>1} \frac{1}{p}, \quad \sum_{|f(p)| \leq 1} \frac{f(p)}{p}, \quad \sum_{|f(p)| \leq 1} \frac{f(p)^{2}}{p}
$$

converge. The latter is the classical condition of Erdös and Wintner required when considering frequencies over the natural numbers [7]. More complicated examples involving unbounded renormalisations of additive functions can also be successfully treated.

The method of this paper lends itself well to the study of the representation of rationals by products and quotients of shifted primes.

The proofs of Theorems 1 and 2 apply Fourier analysis. Since the Féjer kernel is nonnegative, $Q_{h}$ does not exceed

$$
3 \pi(x)^{-1} \sum_{p \leq x} \int_{-1}^{1}(1-|t|) e^{-i t h} g(p+a) d t,
$$

where $g(n)$ is the multiplicative function $\exp (\operatorname{it} f(n))$. To deal directly with the mean value of $g(p+a)$ over the primes would require finer information 
concerning the distribution of primes in residue classes than is currently available. Let $3|a| \leq w \leq z$. Ultimately $z$ will be chosen a power of $x, w$ a power of $\log x$. Let $P, R$ denote the products of the primes in the ranges $3|a|<p \leq w, w<p \leq z$ respectively. I majorize (1) by introducing a Selberg square function $\left(\sum_{d \mid(n, R)} \lambda_{d}\right)^{2}$, where the $\lambda_{d}$ are real, zero if $d>z, \lambda_{1}=1$. Expanding and interchanging the order of summation gives

$$
Q_{h} \leq \frac{3}{\pi(x)} \int_{-1}^{1}(1-|t|) e^{-i t h} \sum_{d_{j} \mid R} \lambda_{d_{1}} \lambda_{d_{2}} \sum_{\substack{n \leq x,(n, P)=1 \\ n \equiv 0\left(\bmod \left[d_{1}, d_{2}\right]\right)}} g(n+a) d t+\frac{3 z}{\pi(x)}
$$

We are reduced to the study of multiplicative functions on arithmetic progressions with moduli large compared to $x$. It may seem curious to retain the condition $(n, P)=1$. However, the choice of a nonprincipal character (mod 3) for $g$ shows that the expected estimate

$$
\sum_{\substack{n \leq x \\ n \equiv r(\bmod D)}} g(n)=\frac{1}{\phi(D)} \sum_{\substack{n \leq x \\(n, \bar{D})=1}} g(n)+\text { 'small' }
$$

is in general false. In $[1,4,6]$ it is shown that the moduli $D$ for which such an estimate fails to be reasonably true are multiples of a single modulus $D_{0}$. The present situation is arranged so that the complications due to the existence of $D_{0}$ are bound up in the condition $(n, P)=1$ and that effectively $D_{0}, R$ have no common divisors.

The moduli $d_{j}$ dividing $R$, with $d_{j} \leq z$ are dealt with by means of the following result.

For a multiplicative function $g$, with values in the complex unit disc, define an exponentially multiplicative function $g_{1}$ by $g_{1}\left(p^{k}\right)=g(p)^{k} / k$ !. Define the multiplicative function $h$ by convolution: $g=h * g_{1}$. Thus $g(p)=g_{1}(p)$, $h(p)=0$. Moreover, $\left|h\left(p^{k}\right)\right| \leq e$. For $A \geq 0$ define

$$
\begin{aligned}
& \beta_{1}(n)=\sum_{\substack{u m p=n \\
u \leq(\log x)^{2 A} \\
p \leq(\log x)^{6 A+15}}} h(u) g_{1}(m) g(p) \frac{\log p}{\log m p}, \\
& \beta_{2}(n)=\sum_{\substack{u r p=n \\
u \leq(\log x)^{2 A} \\
r \leq(\log x)^{6 A+15}}} h(u) g_{1}(r) g(p) \frac{\log p}{\log r p},
\end{aligned}
$$

and set $\beta(n)=g(n)-\beta_{1}(n)-\beta_{2}(n)$. Note that $\beta_{j}(n) \ll 1$ uniformly in $n, j$.

Lemma 1. Let $0<\delta<1 / 2$. Then

$$
\begin{aligned}
& \sum_{D_{1} D_{2} \leq x^{\delta}} \max _{\left(r, D_{1} D_{2}\right)=1} \max _{y \leq x}\left|\sum_{\substack{n \leq y \\
n \equiv r\left(\bmod D_{1} D_{2}\right)}} \beta(n)-\frac{1}{\phi(D)} \sum_{\substack{n \leq y,\left(n, D_{2}\right)=1 \\
n \equiv r\left(\bmod D_{1}\right)}} \beta(n)\right| \\
& \ll x(\log x)^{-A}(\log \log x)^{2}+w^{-1} x(\log x)^{2 A+8}(\log \log x)^{2} \\
& +w^{-1 / 2} x(\log x)^{5 / 2} \log \log x,
\end{aligned}
$$


where $D_{1}$ is confined to integers whose prime factors do not exceed $w$ and $D_{2}$ to integers all of whose prime factors exceed $w$. The implied constant depends at most upon $\delta, A$.

Lemma 1 represents a generalisation to largely arbitrary multiplicative functions of the well-known theorem of Bombieri and Vinogradov concerning primes in arithmetic progressions. The parameter $\delta$ may be replaced by $1 / 2-\varepsilon(x)$ for a certain positive function $\varepsilon(x)$, which approaches zero as $x \rightarrow \infty$. Of importance here is the quality of the error term. For $w \geq(\log x)^{3 A+8}$ it is as good as that of Bombieri and Vinogradov. To this end the functions $\beta_{j}$ were introduced, manifesting the assertion of $[5$, p. 408], already in view in $[3, p$. 178], that for general multiplicative functions a change of form would be required. In particular, $\beta_{2}(n)$ is largely supported on the primes and cannot be removed without further information concerning $g$. Most integers $n$ will have few prime divisors, so that effectively the $\beta_{j}(n)$ are $\ll \log \log x / \log x$ over the range $2 \leq n \leq x$.

The functions $\beta_{j}$ run through the treatment of the integral at (2) along with the central function $g$. A notable feature of the method is the casting of the Selberg square functions on the multiplicative integers in a rôle, which on the additive group of reals, is traditionally played by a Féjer kernel. The outcome is the estimate

(4) $Q_{h} \ll x^{-1} \log w \int_{-1}^{1}(1-|t|) e^{-i t h} \sum_{\substack{n \leq x \\(n, \bar{P})=1}} g(n+a) d t+(\log x)^{-1}(\log \log x)^{2}$.

The complications introduced by the exceptional modulus $D_{0}$ mentioned earlier must now be dealt with. To this end [4] or [6] may be applied. For simplicity of exposition I appeal to Theorem 1 of [6].

Lemma 2. Let $0<\gamma<1,0<\delta<1 / 8,2 \leq \log N \leq Q \leq N$. Then any multiplicative function $g$ with values in the complex unit disc satisfies

$$
\sum_{\substack{n \leq x \\ n \equiv r(\bmod D)}} g(n)=\frac{1}{\phi(D)} \sum_{\substack{n \leq x \\(n, D)=1}} g(n)+O\left(\frac{x}{\phi(D)}\left(\frac{\log Q}{\log x}\right)^{1 / 8-\delta}\right)
$$

uniformly for $N^{\gamma} \leq x \leq N$, for all $(r, D)=1$, for all $D \leq Q$ save possibly for the multiples of a $D_{0}>1$.

From Lemma 2 with $N=x, Q=\exp \left((\log \log x)^{2}\right)$ I obtain the following estimate.

Lemma 3. Let $w$ be a power of $\log x$ and $P$ the product of the primes in the interval $(y, w]$, where $3|a| \leq y \leq w$. Then either

$$
\sum_{\substack{n \leq x \\(n-a, P)=1}} g(n)=\prod_{y<p \leq w}\left(1-\frac{1}{p-1}\right) \sum_{n \leq x} g(n) \prod_{\substack{p \mid n \\ p>y}}\left(\frac{p-1}{p-2}\right)+O\left(x(\log x)^{-1 / 10}\right),
$$

or there is a prime divisor $q$ of $P$ such that

$$
\begin{aligned}
\sum_{\substack{n \leq x \\
(n-a, P)=1}} g(n)= & \prod_{\substack{y<p \leq w \\
p \neq q}}\left(1-\frac{1}{p-1}\right) \sum_{\substack{n \leq x \\
(n, q)=1}} g(n) \prod_{\substack{p \mid n \\
p>y}}\left(\frac{p-1}{p-2}\right) \\
& +O\left(x(\log x)^{-1 / 10}\right) .
\end{aligned}
$$


The implied constants do not depend upon $y, g$, or $q$.

The prime $q$ may vary with $g$ and $x$.

It follows from (4) and Lemma 3 that

$$
Q_{h} \ll x^{-1} \int_{-1}^{1}(1-|t|) e^{-i t h} \sum_{n \leq x} g(n) \prod_{\substack{p|n \\ p>3| a \mid}}\left(\frac{p-1}{p-2}\right) d t+(\log x)^{-1 / 12},
$$

with possibly a condition $(n, q)=1$ required in the sum. Whilst the function $g$ in Lemmas 1, 2, and 3 may be arbitrary up to having values in the unit complex disc, in (5) $g$ has the special form $\exp (i t f(n))$. The exceptional prime $q$ may therefore vary with $t$. It can be arranged that $q$ may only exist on intervals, on each of which it will be constant. The integral at (5) is therefore well defined. Without the condition $(n, q)=1$ we may now follow the original treatment of Ruzsa [9], who considered a similar integral without the weight factor $\Pi(p-1) /(p-2)$. The extra condition $(n, q)=1$ introduces some further complications, but they can be overcome.

Similarly

$$
S_{h} \ll \phi(N)^{-1} \int_{-1}^{1}(1-|t|) e^{-i t h} \sum_{\substack{n \leq N \\(n, N)=1}} g(n) \prod_{\substack{p \mid n \\ p>3}}\left(\frac{p-1}{p-2}\right) d t+(\log N)^{-1 / 10} .
$$

Once again an auxiliary condition $(n, q)=1$ may be needed in the sum. Since $N$ may have many prime factors, the condition $(n, N)=1$ introduces a new complication, but this, too, can be overcome. It may be remarked here that Theorem 2 of [2] shows that in quite general circumstances conditions of the type $(n, N)=1$ may be factored out of mean values of multiplicative functions.

It transpires that the parameter $\lambda$ appearing in the definitions of $W(x)$ and $Y(N)$ may be restricted by $|\lambda| \leq(\log x)^{2},|\lambda| \leq(\log N)^{2}$ respectively.

\section{REFERENCES}

1. P.D.T.A. Elliott, Multiplicative functions on arithmetic progressions, Mathematika 34 (1987), 199-206.

2. __ Extrapolating the mean-values of multiplicative functions, Nederl. Akad. Wetensch. Proc. Ser. A 92 (1989), 409-420.

3. _ Multiplicative functions on arithmetic progressions III: The large moduli, A tribute to Paul Erdös (A. Baker, B. Bollobás, and A. Hajnal, eds.), Cambridge Univ. Press Cambridge, 1990, pp. 177-194.

4. __ Multiplicative functions on arithmetic progressions IV: The middle moduli, J. London Math. Soc. (2) 41 (1990), 201-216.

5. __ Multiplicative functions on arithmetic progressions V: Composite moduli, J. London Math. Soc. (2) 41 (1990), 408-424.

6. __ Multiplicative functions on arithmetic progressions VI: More middle moduli, J. Number Theory (to appear).

7. P. Erdös and A. Wintner, Additive arithmetical functions and statistical independence, Amer. J. Math. 61 (1939), 713-721.

8. G. Halász, On the distribution of additive arithmetical functions, Acta Arith. 27 (1975), 143-152. 
9. I. Z. Ruzsa, On the concentration of additive functions, Acta Math. Hungar. 36 (1980), 215232.

10. N. M. Timofeev, The Erdös-Kubilius conjecture concerning the value distribution of additive functions on the sequence of shifted primes, Acta Arith. LVIII (1991), 113-131. (Russian)

DePartment of Mathematics, University of Colorado, Boulder, Colorado 80309-0001 